\title{
Electron emission: From the Fowler-Nordheim relation to the Child-Langmuir law
}

\author{
Y. Y. Lau and Youfan Liu \\ Intense Energy Beam Interaction Laboratory and Department of Nuclear Engineering, \\ University of Michigan, Ann Arbor, Michigan 48109-2104 \\ R. K. Parker \\ Vacuum Electronics Branch, Electronics Science and Technology Division, \\ Naval Research Laboratory, Washington, DC 20375
}

(Received 9 September 1993; accepted 14 February 1994)

\begin{abstract}
Universal voltage-current characteristics are presented for a planar diode, showing the general transition from the Fowler-Nordheim relation to the Child-Langmuir law. These curves are normalized to the intrinsic scales that are constructed from the Fowler-Nordheim coefficients $A, B$. They provide an immediate assessment of the importance of the space charge effects, once the gap voltage, gap spacing, and the Fowler-Nordheim coefficients ate specified. An example in the parameter regime of vacuum microelectronics is presented.
\end{abstract}

\section{INTRODUCTION}

The emerging field of vacuum microelectronics ${ }^{1-4}$ offers exciting possibilities in miniature coherent radiation sources, ${ }^{5}$ flat pancl displays, ${ }^{6}$ and ultrafast logic circuits. ${ }^{7}$ The crucial component is the electron emitter, which consists of an array of sharp tips fabricated by lithographic techniques. ${ }^{8}$ Some of these tips may have a radius of curvature down to tens of angstroms and a packing density reaching $10^{9}$ tips $/ \mathrm{cm}^{2}$ (Ref. 9). When subjected to an external voltage, field emission occurs on these tips as the surface electric field $E_{s}$ there easily exceeds $10^{9} \mathrm{~V} / \mathrm{m}$. The emitted current density is determined by the Fowler-Nordheim (FN) equation, ${ }^{10}$ which in its simplest approximation is described by the FN coefficients $A, B$ [defined in Eq. (5) below].

In some sense, this emission is self-regulating. As the surface electric field increases, the emission current increases, leading to a charge buildup which in turn limits the surface electric field. In fact, when this happens, the space charge effects become important and we expect that the voltage current $(V-I)$ characteristics turn into the ChildLangmuir law. ${ }^{11}$ Note that the Fowler-Nordheim relation is material dependent whereas the Child-Langmuir law is not. In this paper, we investigate this transition, a problem of considerable current interest ${ }^{1}$ because of the high current densities expected from the sharp tips. Specifically, we construct a set of universal $V-I$ characteristics using a planar model. They are termed "universal" because they are independent of the materials or the work function. This is possible because out of the Fowler-Nordheim coefficients $A, B$, we may construct intrinsic scales to which all physical quantities may be normalized. These characteristics then clearly show the condition under which the space charge effects become important. Given the properties of the emitting material (i.e., given $A, B$ ), the $V-I$ characteristics may be immediately determined once the anode-cathode spacing is specified.

There are related works on the effects of space charge on field emission. A recent one is published by Anderson, ${ }^{12}$ who compares the emission current, with and without the effects of space charge, as a function of the surface electric field. This approach is not too convenient since the surface electric field itself is an unknown quantity. Anderson also considers the cylindrical and spherical geometries. No $V-I$ characteristics are given, however. The $V-I$ characteristics, including the transition, were studied by Babour et al. ${ }^{13}$ who provided an argument for the justification of the use of the planar model to interpret their experiments that employed sharp emitters. Here, we greatly expand the theories in Refs. 12 and 13. Constructing the natural scales based on the properties of the emitting material, we condense the vast parameter space into a single plot (Fig. 3). Quantum mechanical behavior of the eleciron motion within a microscopic diode, including the effects of space charge, was considered in Ref. 14. Other earlier works on thermal instability and on diode closure triggered by sharp emitters are addressed in the last section.

\section{ANALYSIS}

In the one dimensional model, the cathode is located at $x=0$ and the anode at $x=D$, held at voltage $V$ with respect to the cathode. We assume that the electrons are emitted from the cathode with negligible initial velocity. Let $n(x), v(x)$, $\Phi(x), E(x)$ be, respectively, the electron density, electron velocity, the electrostatic potential, and the electric field within the gap. The current density $J=e n v$ is constant, and is assumed to be related to the surface electric field $E_{s}=E(0)$ by the Fowler-Nordheim equation. In this paper, the quantities $e, J, E, \Phi, v, V$ are all positive, by convention.

From conservation of energy, $e \Phi=m v^{2} / 2$, we may write the Poisson equation

$$
\frac{d^{2} \Phi}{d x^{2}}=\frac{e n}{\epsilon_{0}}=\frac{J}{\epsilon_{0} v}
$$

in the Llewellyn form ${ }^{15}$ 


$$
\frac{d^{2} v}{d t^{2}}=\frac{e J}{m \epsilon_{0}}
$$

by a change of variable from $x$ to $t: v=d x / d t$. Equation (2) may be readily integrated to yield

$$
v(t)=\frac{e J}{2 m \epsilon_{0}} t^{2}+\frac{e}{m} E_{s} t,
$$

which shows that, initially, the electrons have zero velocity and are accelerated by the surface electric field $E_{s}$. Equation (3) may be integrated again

$$
x(t)=\frac{e J}{6 m \epsilon_{0}} t^{3}+\frac{e E_{s}}{2 m} t^{2} .
$$

Let $T$ be the transit time of an electron crossing the gap, then at $t=T, x=D$, and $v=(2 \mathrm{eV} / \mathrm{m})^{1 / 2}$. The Child-Langmuir law may be obtained when the current density $J$ is so high that the $E_{s}$ term in both Eqs. (3) and (4) may be neglected. Evaluating Eqs. (3) and (4) at $t=T$, and eliminating $T$ from these two equations, one then obtains the Child-Langmuir law, the familiar relation between $J, V$, and $D$.

In general, Eqs. (3) and (4) give two equations between the three variables $J, E_{s}$, and $T$. (We assume that the gap voltage $V$ and the gap spacing $D$ are given.) The third equation required to solve for $J, E_{s}$, and $T$, is the FowlerNordheim relation, with coefficients $A, B$ (Refs. 10,12, and 13)

$$
J=A E_{s}^{2} e^{-B / E_{s}}=J_{0}\left(\frac{E_{s}}{E_{0}}\right)^{2} e^{-1 /\left(E_{s} / E_{0}\right)} .
$$

Equation (5) determines the emission current density $J$ in terms of the surface electric field $E_{s}$. In writing the last form of Eq. (5), we have constructed from the coefficients $A, B$ an electric field scale $E_{0}$ and a current density scale $J_{0}$ with $^{16}$

$$
E_{0}=B, \quad J_{0}=A B^{2} .
$$

Note that these two scales depend only on the intrinsic properties of the emitting material. From these two scales, we may construct a time scale $\tau=\epsilon_{0} E_{0} / J_{0}$ [cf. Eq. (3)], a length scale $l=e E_{0} \tau^{2} / m$ [cf. Eq. (4)], and a voltage scale $U=l E_{0}$. These are the natural scales ${ }^{17}$ from the mathematical structures of the equations. While their physical meanings are not immediately obvious, their introduction enable us to construct universal voltage-current $(V-I)$ characteristics, as follows.

We first use a bar to designate normalized quantities: $\bar{D}=D / l, \bar{T}=T / \tau, \bar{V}=V / U, \bar{E}=E_{s} / E_{0}, \bar{J}=J / J_{0}$. Then $\bar{T}$ may be solved for from the quadratic polynomial, Eq. (3), in terms of $\bar{V}, \bar{J}, \bar{E}$ :

$$
\bar{T}=\frac{\xi}{\bar{E}} e^{1 / \bar{E}}
$$

where

$$
\xi=-1+\sqrt{1+2(2 \bar{V})^{1 / 2} e^{-1 / E}} .
$$

In obtaining Eq. (7), we have used the normalized Eq. (5)

$$
\bar{J}=\bar{E}^{2} e^{-1 / \bar{E}} \text {. }
$$

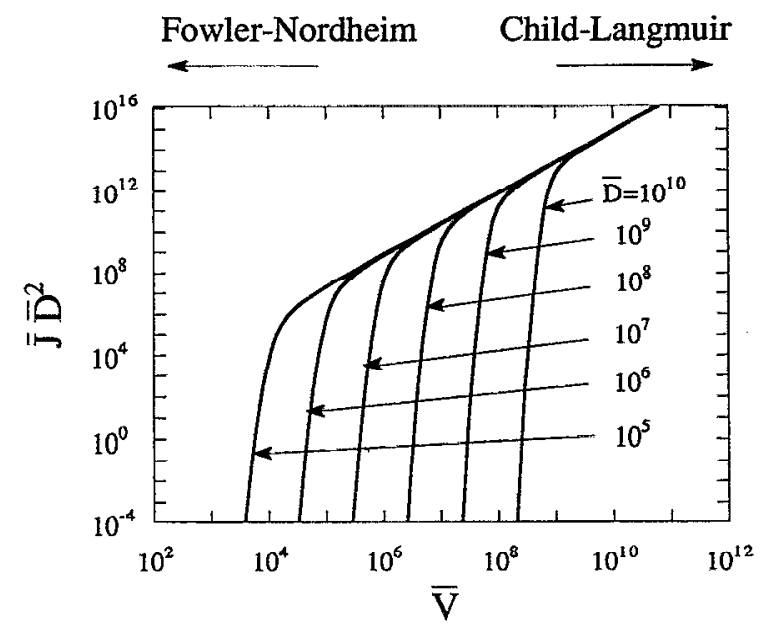

FIG. 1. The normalized $V-I$ characteristics for a field emission diode at various normalized gap spacings $(\bar{D})$. The transition to the Child-Langmuir law at high gap voltages is shown.

Equation (4) then reads

$$
\xi^{2}(\xi+3)=6 \bar{D} \bar{E} e^{-2 / \bar{E}}
$$

upon using Eq. (7).

The universal $V-I$ characteristics may then be constructed as follows. Assume a value of $\bar{D}$. As $\bar{E}$ varies, $\xi$ may be solved for from Eq. (10), a cubic polynomial in $\xi$. From this computed value of $\xi$, Eq. (8) immediately yields $\bar{V}$ as a function of $\bar{E}$ whereas Eq. (9) yields $\bar{J}$ as a function of $\bar{E}$. This last sentence provides the procedure to construct the $V-I$ characteristics using $\bar{E}$ as a running parameter. Shown in Fig. 1 are the $V-I$ characteristics, constructed this way for various values of $\bar{D}$. They are applicable to all materials, as long as the electron emission is governed by Eq. (5).

Each curve in Fig. 1 has two asymptotic behaviors. It approaches the Child-Langmuir law ${ }^{18}$

$$
\bar{J} \bar{D}^{2}=\left(\frac{4 \sqrt{2}}{9}\right)(\bar{V})^{3 / 2}
$$

for large values of $\vec{V}$ at which the space charge effects are important. At lower values of $\bar{V}$, it approaches the FowlerNordheim relation ${ }^{18}$

$$
\tilde{J} \bar{D}^{2}=\bar{V}^{2} e^{-D i V},
$$

which is simply Eq. (9) [or Eq. (5)] in which the surface electric field $E_{s}$ is approximated by the vacuum field $V / D$. Since the Child-Langmuir law is independent of the cathode material, all $V-I$ characteristics will merge into the same asymptote for sufficiently large values of $\vec{V}$, as shown in Eq. (11) and Fig. 1. [See, also, the paragraph following Eq. (4).] It is clear that the space charge effect will become important when Eqs. (11) and (12) yield similar values.

Another way to see the effects of space charge is through a "Fowler-Nordhcim plot," which is a plot of $\log \left(J / V^{2}\right)$ vs $1 / V$. Shown in Fig. 2 is one such plot for the case $\bar{D}=10^{7}$. In this case, the space charge effects become important when $\bar{V} \geqslant 10^{6}$, and are unimportant otherwise. 


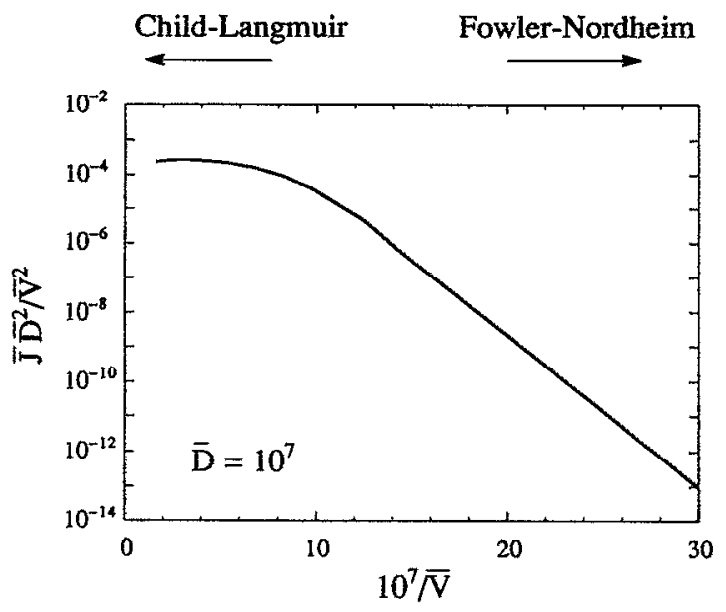

FIG. 2. The Fowler-Nordheim plot for the case $\bar{D}=10^{7}$. The space charge effects are unimportant along the straight line segment of this curve.

As an example, consider an emission model in which the Fowler-Nordheim coefficients $A, B$ are given by [in the standard international (SI) units] ${ }^{12}$

$B=6.49 \times 10^{9} \phi^{1.5}, \quad A=(1.4 / \phi) \times 10^{-6+4.26 / \sqrt{\phi}}$,

where $\phi$ is the work function in electron volts. For an emitter with work function $\phi=2 \mathrm{eV}$, Eq. (6) gives $E_{0}=1.84 \times 10^{10}$ $\mathrm{V} / \mathrm{m}$ and $J_{0}=2.43 \times 10^{17} \mathrm{~A} / \mathrm{m}^{2}$. The other scales are $l=1.45 \times 10^{-15} \mathrm{~m}, U=2.66 \times 10^{-5} \mathrm{~V}, \tau=6.7 \times 10^{-19} \mathrm{~s}$ (Ref. 17). For a gap separation of, say, $1.45 \times 10^{-8} \mathrm{~m}$, then $\bar{D}=10^{7}$. Figure 2 suggests that the space charge effects would dominate if the gap voltage $V>26.6 \mathrm{~V}$ (i.e., $\bar{V}>10^{6}$ ) for this example. Note that at the transition voltage of $26.6 \mathrm{~V}$, the average electric field within the gap is of order 26.6 $\mathrm{V} /\left(1.45 \times 10^{-8} \mathrm{~m}\right)=1.8 \times 10^{9} \mathrm{~V} / \mathrm{m}$, a value within the range expected of field emission.

Transition from the Fowler-Nordheim regime to the Child-Langmuir regime may be said to occur when Eqs. (11) and (12) yield the same value. This happens when

$$
\bar{D}=\bar{V} \ln \left(\frac{9}{4 \sqrt{2}} \sqrt{\bar{V}}\right) .
$$

Equation (14) is plotted in Fig. 3. Note that this curve is material independent.

\section{CONCLUDING REMARKS}

The simple analysis given in this paper omits many physically important aspects, such as the geometrical effects ${ }^{19}$ and the dependence of $A$ and $B$ on the electric field. ${ }^{10,16,19}$ The effect of high surface electric field may also lead to the bending of energy bands in semiconductor emitters. ${ }^{20}$ In the case of very sharp emitters, ${ }^{21-23}$ joule heating of the tip may lead to thermal instability and result in catastrophic destruction of the tips, a phenomenon well documented $^{24,25}$ which may have important implications on the cathode stability and gap closure in field emitter arrays. These are important subjects but are beyond the scope of this

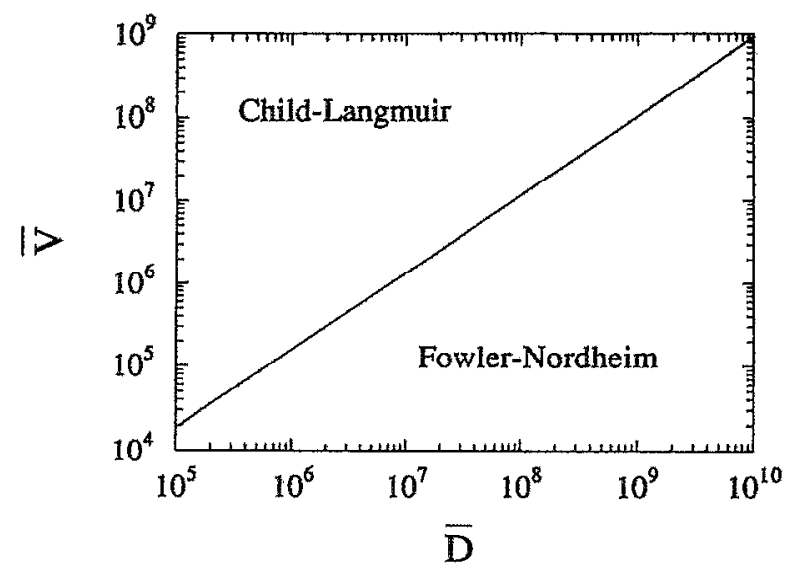

FIG. 3. The universal curve that separates the Fowler-Nordheim regime and the Child-Langmuir regime in a planar diode. This curve is material independent.

paper. Finally, the radio frequency (RF) properties of vacuum microelectronic devices have been explored in terms of the Fowler-Nordheim coefficients. ${ }^{26}$

In summary, we have constructed the universal $V-I$ characteristics that link the Fowler-Nordheim relation to the Child-Langmuir law. These characteristics provide an immediate determination of the importance of the space charge effects, once the emission coefficients $A$ and $B$ [in Eq. (5)], and the gap spacing are given.

\section{ACKNOWLEDGMENTS}

We acknowledge useful discussions with A. K. Ganguly, Henry Gray, Jonathan Shaw, and C. M. Tang.

This work was supported by the Defense Science Office of the Advanced Research Project Agency, by the Naval Research Laboratory, and by the University of Michigan Departmental Funds.

${ }^{1}$ Proceedings of the 6th International Vacuum Microelectronics Conference, Newport, Rhode Island, July, 1993 (Special Issue in J. Vac. Sci. Tech. B 12, 1994). References $2-4$ below are earlier conference proceedings that are similarly published in archival journals.

${ }^{2}$ Special Issue, IEEE Trans. Electron Devices 36 (1989).

${ }^{3}$ Special Issue, IEEE Trans. Electron Devices 38 (1991).

${ }^{4}$ Special Issue, J. Vac. Sci. Technol. B 11 (1993).

${ }^{5}$ R. K. Parker and R. H. Abrams, International Electron Devices Meeting, Technical Digest (Institute of Electrical and Electronic Engineers, New York, 1990), p. 7.1.1 [IEEE Catalog No. 90CH2865-4]; J. P. Calame, H. F. Gray, and J. I. Shaw, J. Appl. Phys. 73, 1485 (1993); W. J. Orvis, C. F. McConagly, D. R. Ciarlo, J. H. Yee, and E. W. Hee, p. 2651 of Ref. 2; N. E. McGruer, A. C. Johnson, S. W. McKnight, W. C. Schwab, C. Chan, and S. Tong, IEEE Trans. ED-38, 666 (1991); C. M. Tang, Y. Y. Lau, and T. Swyden, in Ref. 1

${ }^{6}$ H. F. Gray, Inf. Disp. 9, 9 (1993); J. L. Grand-Clement, in Ref. 1.

${ }^{7}$ I. Brodie, p. 2641 of Ref. 2.

${ }^{8}$ C. A. Spindt, C. E. Holland, A. Rosengreen, and I. Brodie, in Ref. 3, p. 2355, and references cited therein.

${ }^{9}$ C. O. Bozler, in Ref. 1.

${ }^{10}$ R. H. Fowler and L. Nordheim, Proc. R. Soc. London Ser. A 119, 173 (1928); L. Nordheim, ibid. 121, 626 (1928); E. L. Murphy and R. H. Good, Phys. Rev. 102, 1464 (1956). 
${ }^{11}$ C. D. Child, Phys. Rev. 32, 492 (1911); I. Langmuir, ibid. 21, 419 (1923)

${ }^{12}$ W. A. Anderson, in Ref. 4, p. 383.

${ }^{13}$ J. P. Barbour, W. W. Dolan, J. K. Trolan, E. E. Martin, and W. P. Dyke, Phys. Rev. 92, 45 (1953).

${ }^{14}$ Y. Y. Lau, D. Chernin, D. G. Colombant, and P. T. Ho, Phys. Rev. Lett. 66, 1446 (1991).

${ }^{15}$ See, e.g., C. K. Birdsall and W. B. Bridges, Electron Dynamics of Diode Regions (Academic, New York, 1966), p. 30.

${ }^{16} \mathrm{We}$ assume that the coefficients $A, B$ are constants. This need not be trueover a wide range of $E_{s}$. Their values are taken to be the "local values" that are specific to the operating regimes, in which $A, B$ may often be adequately regarded as constants.

${ }^{17}$ Roughly, the current density $J_{0}$ is associated with the supply function; so it is many orders of magnitude higher than the emitted current density. The electric field scale $E_{0}$ is a measire of the surface electric field required to overcome the potential barrier for field emission. The time scale $\tau=\epsilon_{0} E_{0} / J_{0}$ constructed out of these two scales appears to be too short to be associated with the tunneling time through the barrier, even though it is tempting to attach such an interpretation.
${ }^{18}$ If we denote the right-hand side of Eq. (10) by $p$, the cubic polynomial $\xi^{2}(\xi+3)=p$ has the asymptotic solutions: $\xi \rightarrow p^{1 / 3},(p \rightarrow \infty)$; $\xi \rightarrow \sqrt{p / 3},(p \rightarrow 0)$. The Child-Langmuir law (11) is obtained for large $p$, and the Fowler-Nordheim relation (12) is obtained for small $p$.

${ }^{19}$ P. H. Cutler, J. He, N. M. Miskovsky, T: E. Sullivan, and B. Weiss, in Ref. 4, p. 387, and references cited therein; D. A. Kirkpatrick, A. Mankofsky, and K. T. Tsang, Appl. Phys. Lett. 60, 2065, 1556 (1992).

${ }^{20} \mathrm{~K}$. L. Jensen and A. K. Ganguly, in Ref. 4, p. 371.

${ }^{21}$ F. M. Charbonnier, C. J. Bennette, and L. W. Swanson, J. Appl. Phys. 38, 627, 634 (1967).

${ }^{22}$ H. E. 'lomaschke and D. Alpert, J. Appl. Phys, 38, 881 (1967); J. Vac. Sci. Tech. 4, 192 (1967).

${ }^{23}$ T. Utsumi, J. Appl. Phys. 38, 2989 (1967).

${ }^{24}$ S. P. Bugayev; G. A. Mesyats, and D. I. Proskurovskii, Sov. Phys. Dokl. 14, 605 (1969).

${ }^{25}$ R. K. Parker, R. E. Anderson, and C. V. Duncan, J. Appl. Phys. 45, 2463 (1974).

${ }^{26}$ J. P. Calame, H. F. Gray, and J. L. Shaw, J. Äppl. Phys. 73, 1485 (1993). 